\title{
An investigation of non-destructive thermographic inspection exploiting phase transition of water for moisture detection in aircraft structures
}

\author{
Eetta Saarimäki*, Peter Ylinen**
}

\author{
* VTT Technical Research Centre of Finland, Tampere, Finland \\ ${ }^{* *}$ FiAF Finnish Air Force, Pirkkala, Finland
}

\section{Abstract}

In this work, thermographic investigation is done to find a suitable procedure to find penetrated water from the composite aircraft structures. This work is concentrating on the inspection method, where the whole structure is cooled below the freezing point of the water and then warmed into the room temperature. Phase transition energy, which is needed to melt the penetrated water, can be detected by thermography, either as an anomaly in individual thermocraphic images, or as a plateau in the thermocraphic monitoring curve.

Author keywords: Composites, thermal NDI, phase transition of water

\section{Introduction}

Thermographic non-destructive inspection methods have been widely researched and defect detection methods developed for aviation purposes, because they can be used without removing aircraft composite parts from the aircraft and because the possibility to investigate large areas simultaneously $[1,2,3,4,5]$. Large area inspection without removing parts from the aircraft reduces the maintenance and inspection time significantly. Recently has been reported an approach to exploit energy needed for phase transition of water in thermographic inspection $[6,7]$.

Penetrated water in the composite sandwich structures can cause problems, because moisture corrodes the honeycomb and further reduces the strength of the adhesive. Water can also cause additional defects during the composite repairs, which have resulted because of the expansion of the moisture (in closed cavity), hence causing skin blow core phenomena during the curing cycle (heating) of the repair.

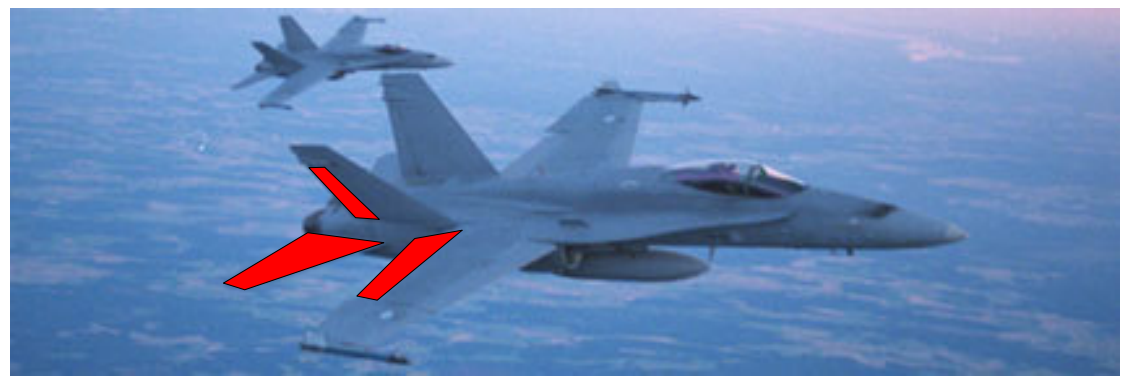

Fig. 1. Ther moisture detection areas under investigation (port and starboard side of the aircraft). Red indicates the interest area of flight control surfaces (rudder, trailing edge flap and horizontal stabilizer). Courtesy of the FiAF.

Thermographic research has continued for several years in close cooperation between VTT and the FiAF $[7,8]$. Recently the main effort has been put on finding a reliable testing conditions and routines for the inspection method to detect penetrated moisture in real aircraft parts (Fig. 1).

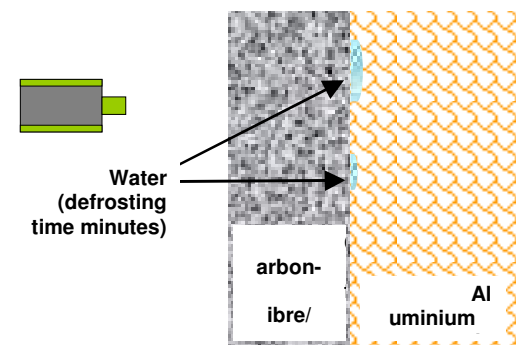

Fig. 2. The principle of the thermographic inspection based on the phase transition of water. 
Thermographic inspection based on the phase transition of water exploits the phase transition energy that is needed for the water defrosting (melting) (Fig. 2). Advantage of this method is that no additional excitation source is needed for the tests. Method based on phase transition can be especially exploited during the long period of arctic weather conditions in Finland and other cold areas. Whole aircraft, or separated parts of the aircraft, can be placed in frozen conditions for few hours. Moisture or penetrated water can be detected, because bigger amount of specific heat of water is needed in the defrosting than warming up the surrounding structure. Aircraft can be either inspected right after a flight [2], or it can be left outside in freezing conditions overnight and inspected when it has been brought in to the maintenance hall to warm conditions [7].

\section{Equipment}

Thermographic measurements were performed with FLIR ThermaCam ${ }^{\mathrm{TM}}$ S65 equipment (detector type Focal Plane Array (FPA), uncooled microbolometer. 320*240 pixels). Thermal sensitivity of the equipment is 0.08 ${ }^{\circ} \mathrm{C}$ with the maximum image frequency of $60 \mathrm{~Hz}$ (using FireWire output and a PC). Artificial defect was measured with Agema 900 LW equipment (detector type MCT, stirling cooled, $272 * 136$ pixels). Thermal sensitivity of the equipment is $0.08{ }^{\circ} \mathrm{C}$ with the maximum image frequency of $50 \mathrm{~Hz}$

Additional measurements, to insure the equal location of the measurement points between different climate conditions, were performed as thermo element measurements with two NI data logger 9211 measurement units each measuring 4 channels.

Structures were cooled either in climate room or outside in freezing conditions during the cold winter season.

\section{Experiments and results}

\subsection{Artificial test sample}

Small amounts of water $(0.1$ and $0.7 \mathrm{~g})$ were injected into the laminate honeycomb (carbon fibre/epoxide composite with aluminium honeycomb core) interface. Artificial test sample was placed into a freezer $-20^{\circ} \mathrm{C}$ for several hours and then returned to room temperature $\left(22^{\circ} \mathrm{C}\right)$. Thermographic inspection was performed at $0.1 \mathrm{~Hz}$ frequency. Phase transition of water can be seen in defected areas as a plateau in the inspection curve (Fig. 3). After the melting of the frozen water the surface temperature of the defected area starts to reach the temperature of the surrounding non-defected area.

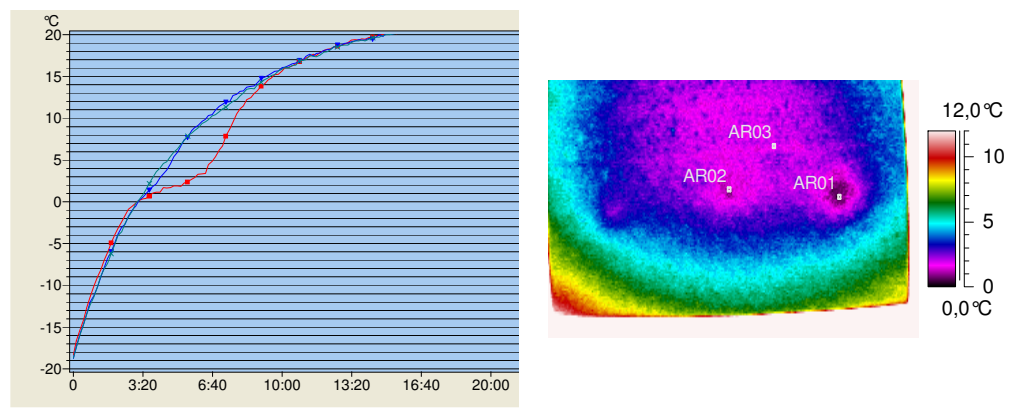

Fig. 3. Penetrated water (in laminate/honeycomb interface) detected with thermographic inspection based on phase transition of water. AR01 refers to red line $(0.7 \mathrm{~g}$ water), AR02 refers to blue line $(0.1 \mathrm{~g}$ water) and AR03 is a reference of dry area (green).

Small amounts of penetrated water can only be seen for a very short period of time. $0.1 \mathrm{~g}$ of water under carbon fibre/epoxide laminate was hardly detectable and would have easily disappeared to variation of the surface temperature in real structure. Less than $1 \mathrm{~g}$ of water can be detected, both from the curve and from the thermographic image, during the first few minutes after the non-defected area has reached $0{ }^{\circ} \mathrm{C}$.

\subsection{Effect of initial climate conditions (rudder warming)}

Research was continued with real aircraft structures. Real structures are commonly more complicated due to the (metallic) supporting structures and/or changes in material thicknesses inside the structure. Sufficient amount of reference data (inspected non-defected structures) was found to be a very practical way of defining warming-up behaviour of non-defected structures.

Speed of the warming-up process of the real rudder structure was inspected, in order to find proper optimal inspection frame (OIF) time for finding the penetrated water from the structure. Determination of OIF-time ensures that possible penetrated moisture is visible at the time of survey, even if only single images were taken from the desired area. Critical parts of the rudder are assumed to be around hinges, due to the possible leak in the sealing of the grounding grooves. Therefore main effort of seeking moisture from the structure was 
concentrated to these areas (and reference point was set near the upper hinge). Optimal inspection frame time was also determined to the whole structure.

According to the inspections with artificial defects, the optimal temperature frame to detect water penetration exploiting phase transition of water, is when the temperature of the surrounding non-defected area is from 5 to $9{ }^{\circ} \mathrm{C}$. (Large amount of water can be detected even at higher temperatures.) Thermographic inspections were ensured with thermoelement measurements to ensure the equal measuring points for each measurement (Fig. 4). The effect of different climate conditions was inspected in four different initial temperatures $(-20,-15,-10$, and $-5{ }^{\circ} \mathrm{C}$ ) and two different cooling times $(3 \mathrm{~h}$ and overnight) with real rudder structure. After cooling down the rudder was returned to the room temperature $\left(22^{\circ} \mathrm{C}\right)$ and the speed of the warming-up process was measured in each case. Warming-up of the composite structure (carbon fibre/epoxide composite with aluminium honeycomb core) under zero degrees was very rapid, thus the reference point to compare optimal inspection time (time frame) of different climate conditions (i.e. initial temperatures) was selected to $0{ }^{\circ} \mathrm{C}$.
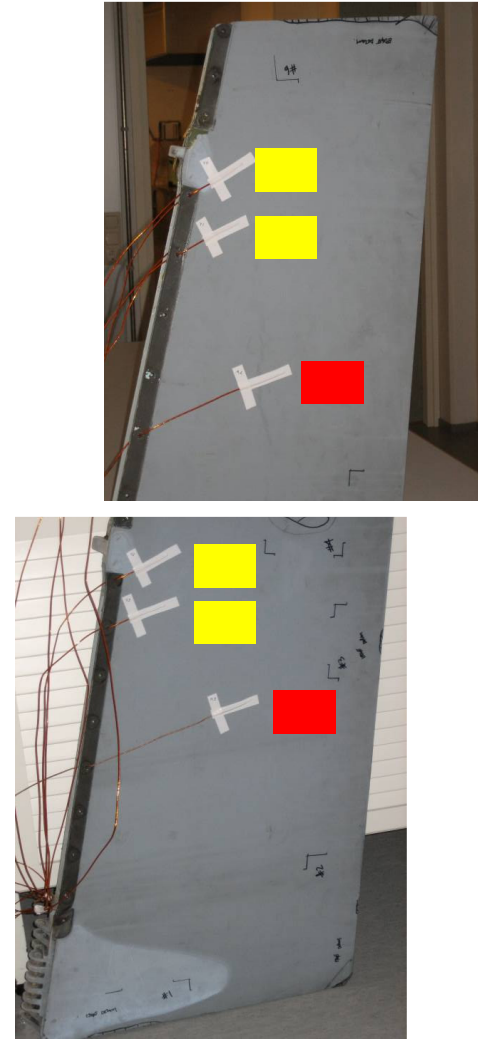

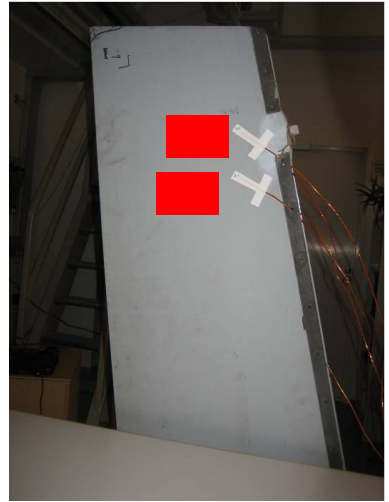

Measuring points of the thermoelements in rudder inspection

Fig. 4. Inspected rudder and the measuring points of the thermoelements. Thermographic inspection performed on the front surface (equals thermoelement points $Y 0-Y 3$ and $A 2-A 3$ ).

Exploitation of phase transition of water needs proper cooling of the structure. Fig. 5 shows that all measured points had reached the cooling temperature $\left(-5^{\circ} \mathrm{C}\right)$. Measuring points $\mathrm{Y} 0 / \mathrm{A} 0$ and $\mathrm{Y} 1 / \mathrm{A} 1$ were placed to the opposite sides of the same rudder structure. Warming-up of the points $Y 0$ and $A 0$ are logical to the nondefected structure. Warming-up of the $Y 1$ and $Y 2$ showed very small plateau at the curve, referring to the moisture inside the rudder. Penetrated moisture could equally be seen in thermographic images taken during the inspection (Fig. 6). 


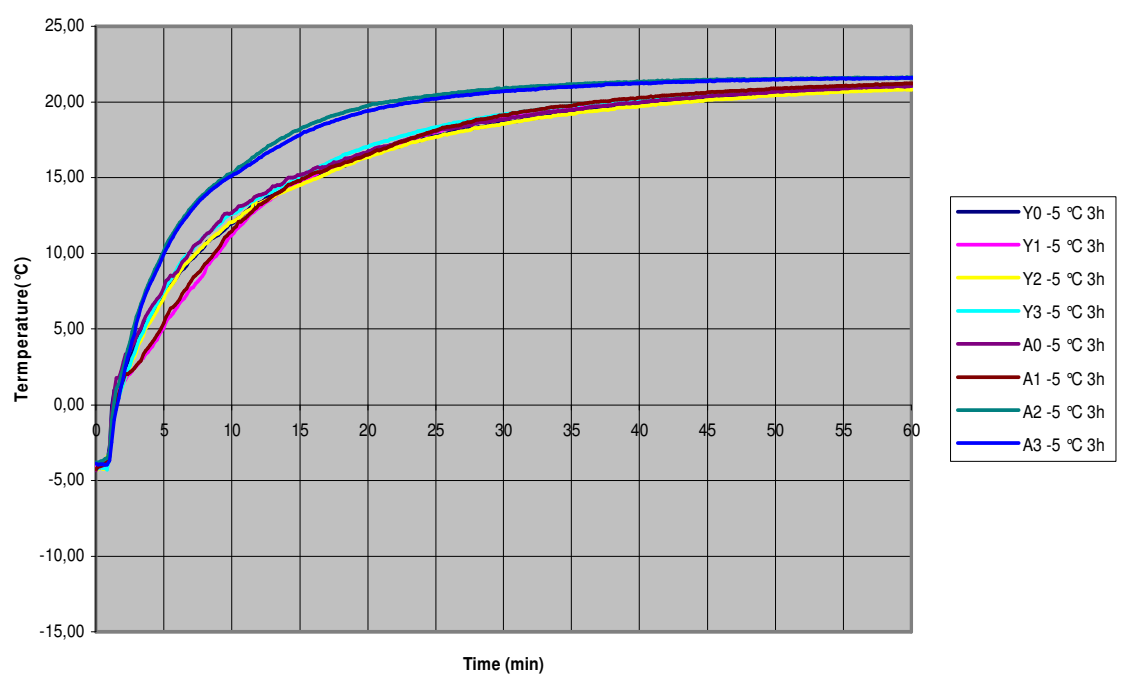

Fig. 5. Comparison of the temperature evolution of all measurement points of the rudder. Initial temperature $-5{ }^{\circ} \mathrm{C}$, cooling time 3 hours.

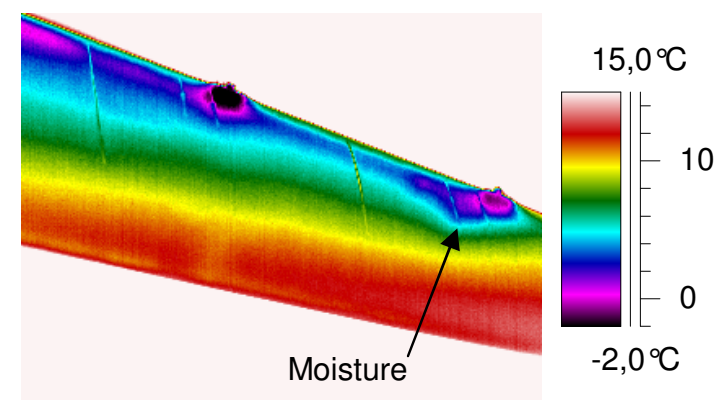

Fig. 6. Moisture detected under the upper hinge of the rudder test sample.

Comparison of the temperature evolution in different climate conditions seemed to be quite equal in the near area of upper hinge (Y0). However, a closer study showed that the optimal inspection frame (OIF) time was from 2-5 minutes to 3-7 minutes after $0{ }^{\circ} \mathrm{C}$ was received (Fig. 7 ).

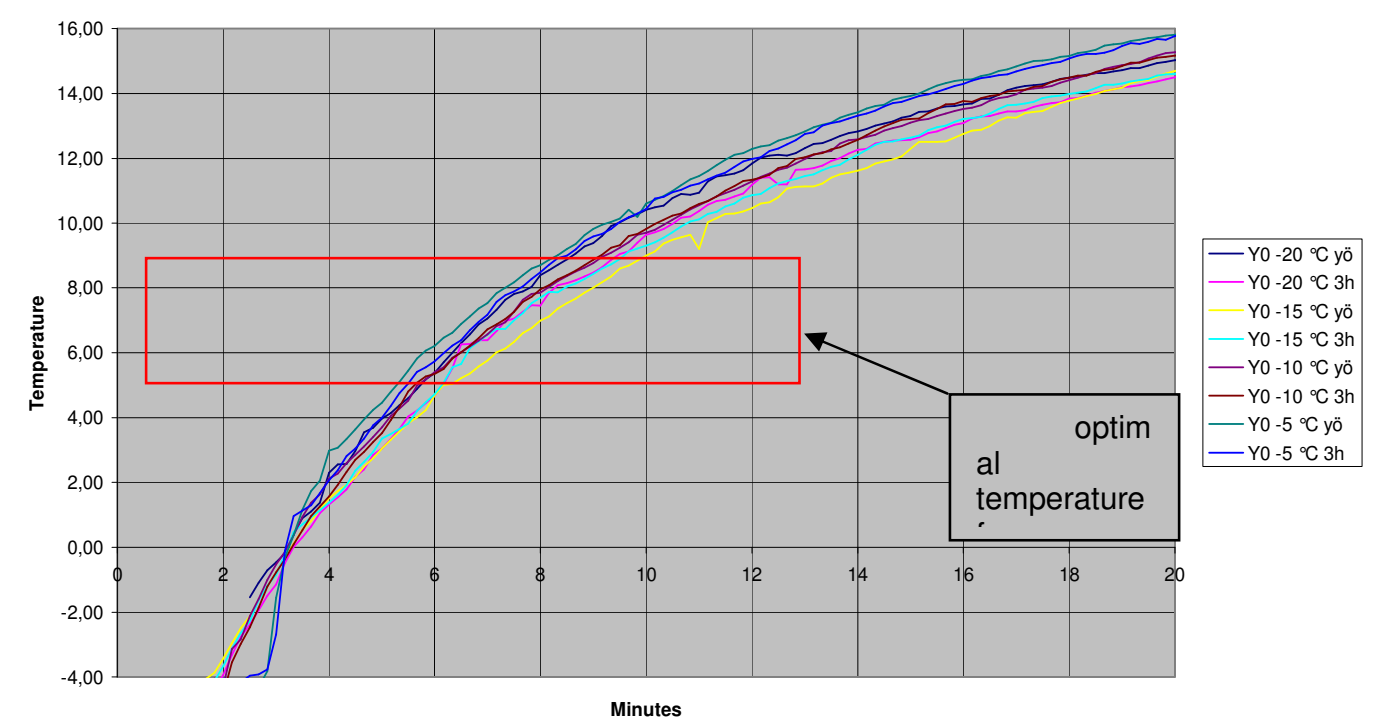

Fig. 7. Comparison of temperature evotution of point YO near uppre hinge of the rudder. Initial temperatures 20, $-15,-10$ and $-5{ }^{\circ} \mathrm{C}$, cooling times 3 hours and over night (=yö.) 
Optimal inspection frame time for the rudder structure was determined with isotherm tool by setting the isotherm temperature range from 5 to $9{ }^{\circ} \mathrm{C}$. An example is taken from a rudder inspection where initial temperature was $-15^{\circ} \mathrm{C}$ and cooling time 3 hours. Zero time was set to the point were the first edge of the inspected rudder reached $5{ }^{\circ} \mathrm{C}$ (Fig. 8, 0 minutes).
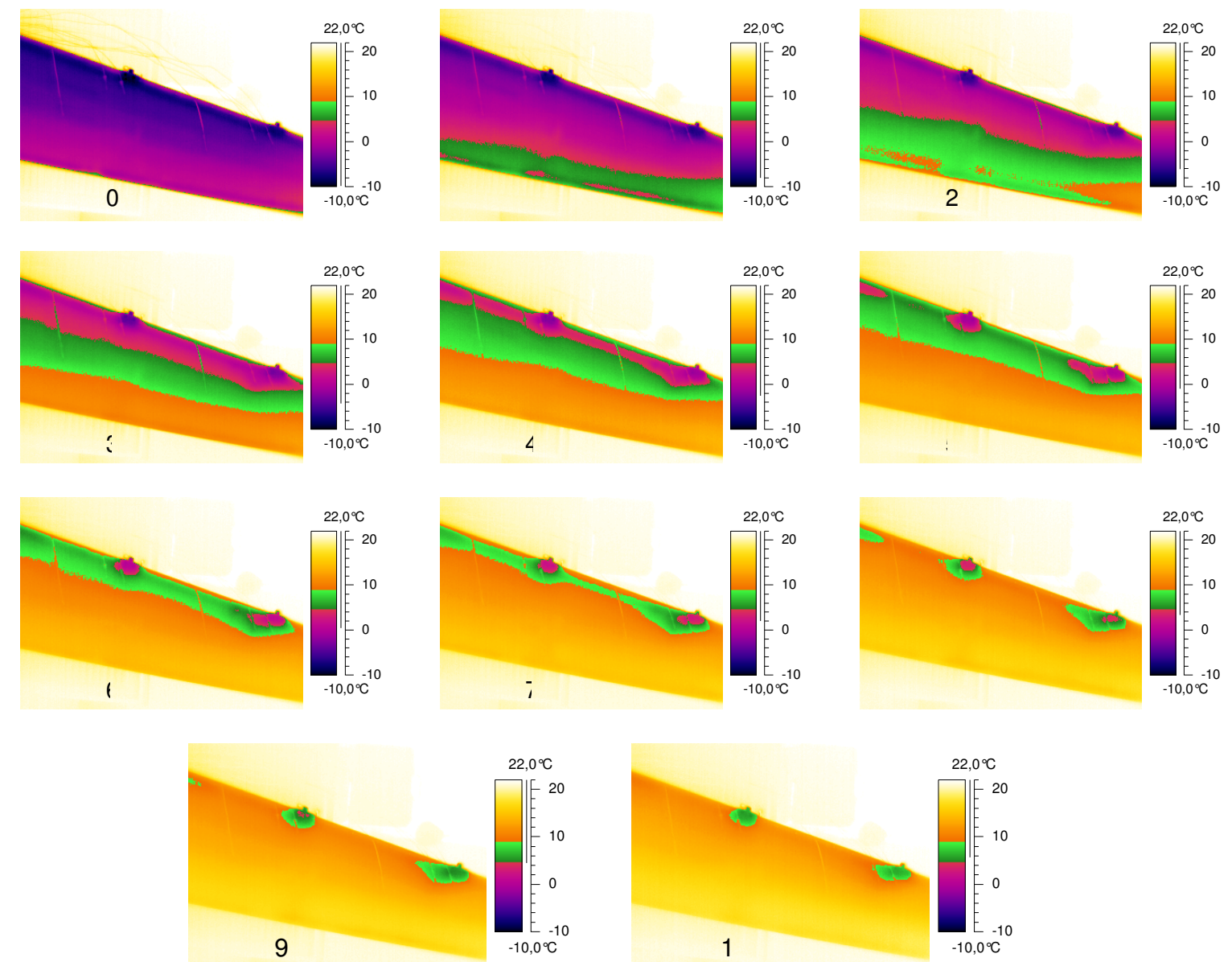

Fig. 8. Optimal inspection frame time for different areas of the rudder surface (green area in the images). 0 time refers the temperature where the first area reaches $5^{\circ} \mathrm{C}$.

Optimal inspection frame time images start to show abnormal warming behaviour around the upper hinge (on the right in the thermographic image) after three minutes of the set zero time (first edge reaches $5^{\circ} \mathrm{C}$ ) (Fig. 8). Individual images do not explain reliably the reason for the abnormal behaviour, but they show clearly the area of interest to be further inspected as a sequence of thermographic images (e.g. real-time monitoring by pulsed thermography or thermography exploiting phase transition of water), or with other NDT-methods sensitive to moisture penetration.

\subsection{Temperature monitoring of multiple flight surfaces}

Further studies concentrated on temperature monitoring of multiple flight - surfaces - rudder, trailing edge flap and horizontal stabilizer. Target of the inspection was to find out the optimal inspection frame time (OIF) for each interest area, by taking individual pictures from each surface. Reference point was selected from the rudder (near upper hinge) and the calculation of the warming time was started as soon as this point reached $0{ }^{\circ} \mathrm{C}$.

The idea to take only individual images of each flight surface during one inspection route came from the fact that it is not economically feasible either to invest to multiple thermographic equipment to follow the temperature evolution of each interesting flight surface, or to cool the whole aircraft for multiple times to inspect each flight surface in a separate inspection route. 

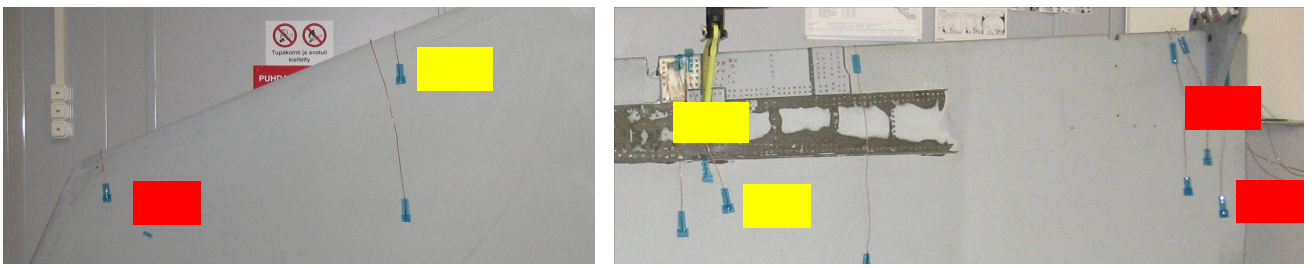

Fig. 9. Monitored points to evaluate optimal inspection frame time (OIF). a) horizontal stabilizer b) Trailing edge flap (monitored around the hinges)

Horizontal stabilizer and trailing edge flap were cooled under zero point outside overnight. Temperature evolution was monitored and compared to the data received from rudder inspections in climate room (Fig. 9 and Fig. 4).

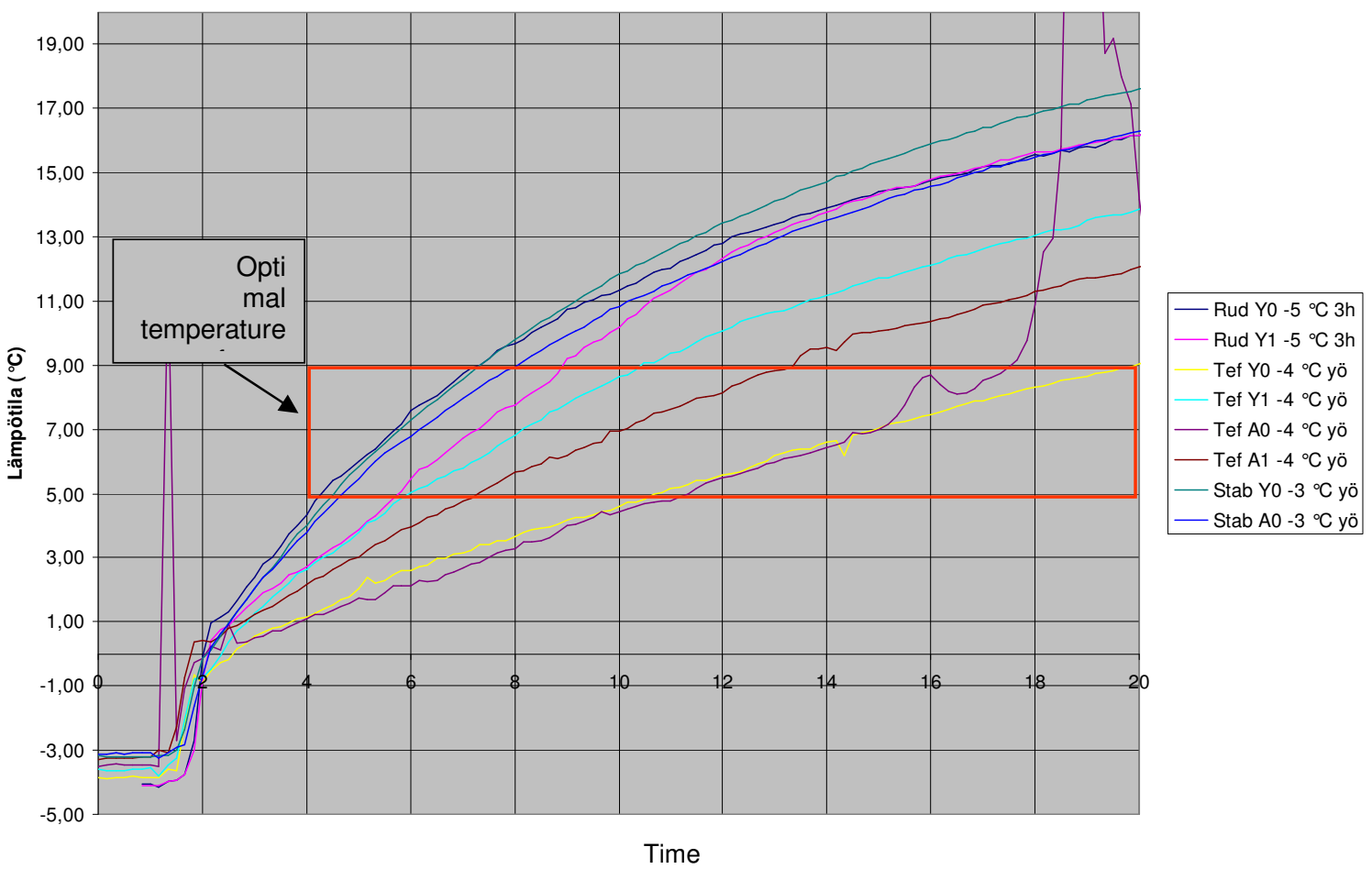

Fig. 10. Comparison of temperature evolution of rudder, horizontal stabilizer and trailing edge flap.

Even though the basic structure of the composites is very similar in all inspected flight surfaces, temperature evolution vary a lot due to the supporting structures close to the monitoring points. If moisture detection of upper rudder hinge is not taken into consideration (Fig. 10, Rud Y1), speed of the warming-up process of the stabilizer and rudder (initial temperature -3 to $-5{ }^{\circ} \mathrm{C}$ ) are almost equal, and optimal inspection frame time is 2 to 6 minutes after the reference point has reached $0{ }^{\circ} \mathrm{C}$. This means that simultaneous inspection of individual images should be taken within 4 minutes for 6 flight surfaces (rudder front and back and stabiliser from the bottom surface from both port and starboard side of the aircraft). Warming-up of the trailing edge flap surface is slower compared to rudder and stabilizer. Areas closest to the hinges take 9 minutes to reach the optimal inspection temperature and can be monitored 9 minutes onwards (Fig. 10).

In order to insure the optimal inspection frame time, measurements have to be done also for the structures that are assembled to the aircraft due to the cooling effect of a fuselage. This phenomenon can be seen around the hinges, and especially in trailing edge flap monitoring points near the fuselage.

\section{Conclusions}

Thermographic inspection based on the phase transition of water has proved to be very sensitive to the moisture. Artificial defected areas showed that very small amounts (less than $1 \mathrm{~g}$ ) of water, in the laminate honeycomb (carbon fibre/epoxide composite with aluminium honeycomb core) interface, can be detected by exploiting phase transition of water in the thermographic inspection.

Multiple tests, with different specimen and different amounts of water, showed that penetrated water can be detected for several minutes as a colder area in the structure. The time and the developed temperature 
difference when the defect can be seen are strongly dependent of the amount of water and the environmental conditions during the inspection.

The effects of environmental conditions were investigated to ensure reliable inspection conditions for the inspected structure. Different cooling temperatures and times were investigated to make sure that the whole structure is frozen before the warming phase. It was shown that it is possible to cool the aircraft parts outdoors in a reliable manner. However different defrosting conditions showed that weather and cooling conditions affected strongly the results.

The optimal observation period to detect small amounts of water was shown to be only few minutes, which leads to the conclusion that defining the optimal inspection frame (OIF) time, to inspect several flight surfaces on one inspection route by taking only individual thermographic images, still needs more inspection with whole aircrafts (parts mounted to the fuselage) in different weather conditions.

\section{Acknowledgments}

Acknowledgments are attributed to FiAF, especially to Air Materiel Command for financial support concerning the thermography research projects and Satakunta Air Command for providing aircrafts to inspection.

\section{REFERENCES}

[1] N.P. Avdelidis, D.P Almond, A. Dobbinson, B.C. Hawtin, C. Ibarra-Castanedo, X. Maldague. Aircraft composites assessment by means of transient thermal NDT, Progress in Aerospace Sciences, 40 (2004) 143-162

[2] VI. Vavilov, A. Klimov, D. Nesteruk, V. Shiryaev. Detecting water in aviation honeycomb structures by using transient IR thermographic NDT, Thermosense XXV, Proceedings of SPIE Vol. 5073 (2003) 345-355

[3] R.W. McCullough, Transient Thermographic Technique for NDI of Aerospace Composite Structures, Thermosense XXVI, Proceedings of SPIE Vol. 5405 (2004) 390-401

[4] T. D'Orazio, C. Guaragnella, M. Leo, P. Spagnolo, Defect detection in aircraft composites by using a neural approach in the analysis of thermographic images, NDT\&E International, 38 (2005) 665-673

[5] N.P. Avdelidis, B.C. Hawtin, D.P. Almond, Transient thermography in the assessment of defects of aircraft composites, NDT\&E International, 36 (2003) 433-439

[6] V. Vavilov, D. Nesteruk. Evaluating water content in aviation honeycomb panels by transient IR thermography, Thermosense XXVII, Proceedings of SPIE Vol. 5782 (2005) 411-415

[7] A. Siljander, E. Saarimäki et al., A review of aeronautical fatigue investigations in Finland during the period May 2005 to April 2007, 30 ${ }^{\text {th }}$ Conference of the International Committee on Aeronautical Fatigue (ICAF), National review 13 (2007) 44-46

[8] A. Siljander, E. Saarimäki et al., A review of aeronautical fatigue investigations in Finland during the period May 2003 to April 2005, $29^{\text {th }}$ Conference of the International Committee on Aeronautical Fatigue (ICAF), National Review (2005) 19,39-40. 
http://dx.doi.org/10.21611/qirt.2008.12_08_16 